\title{
À corps perdu. Limiti, costruzioni e intensità del corpo, a cura di G. ARAGONESE, F. DAINESE, A. NICOLINI, C.
}

\section{VARESCHI}

\section{Gabriella Bosco}

\section{OpenEdition}

\section{Journals}

\section{Edizione digitale}

URL: https://journals.openedition.org/studifrancesi/44334

DOI: 10.4000/studifrancesi.44334

ISSN: 2427-5856

\section{Editore}

Rosenberg \& Sellier

\section{Edizione cartacea}

Data di pubblicazione: 1 juin 2021

Paginazione: 266-268

ISSN: 0039-2944

\section{Notizia bibliografica digitale}

Gabriella Bosco, «À corps perdu. Limiti, costruzioni e intensità del corpo, a cura di G. ARAGONESE, F. DAINESE, A. NICOLINI, C. VARESCHI», Studi Francesi [Online], 193 (LXV | I) | 2021, online dal 01 juillet 2021, consultato il 15 octobre 2022. URL: http://journals.openedition.org/studifrancesi/44334 ; DOI: https://doi.org/ 10.4000/studifrancesi.44334

Questo documento è stato generato automaticamente il 15 octobre 2022.

\section{(i) $\$$

Creative Commons - Attribuzione - Non commerciale - Non opere derivate 4.0 Internazionale - CC BYNC-ND 4.0

https://creativecommons.org/licenses/by-nc-nd/4.0/ 


\title{
À corps perdu. Limiti, costruzioni e intensità del corpo, a cura di $\mathrm{G}$.
} ARAGONESE, F. DAINESE, A. NICOLINI, C. VARESCHI

\author{
Gabriella Bosco
}

\section{NOTIZIA}

À corps perdu. Limiti, costruzioni e intensità del corpo, a cura di G. ARAGONESE, F. DAINESE, A. NICOLINI, C. VARESCHI, Ed. dell'Orso, Alessandria, 2020, «Studi e Ricerche» 180, 367 pp.

1 Un volume ricco, che nasce da un'idea tanto semplice quanto inesauribile: quella di studiare la tematica della corporeità in una prospettiva multidisciplinare, immergendovisi come in una sorta di mise en abyme progettuale, "à corps perdu", all'insegna di un'affermazione tratta dal Mythe de Sisyphe di Albert Camus: "Nous habitons notre corps bien avant de le penser", citata in esergo dai curatori nella loro ampia e articolata Introduzione (pp. 5-15).

2 Ma ricco anche, il volume, in quanto raccolta degli atti di un Convegno internazionale per giovani ricercatori svoltosi a Verona dal 20 al 22 settembre del 2017, ideato da quattro di loro, i curatori del volume, e pensato per dar voce alle ricerche e agli studi di un gran numero di altri giovani ricercatrici e ricercatori, convegno per il quale si deve dir grazie al sostegno e al contributo della Scuola Dottorale di Scienze Umanistiche dell'Università di Verona, diretta all'epoca da Arnaldo Soldani e animato da Giulia ARAGONESE, Francesca DAINESE, Andrea NICOLINI e Carlo VARESCHI con l'ausilio di Annalisa Sacchi, docente di Estetica della scena contemporanea.

3 I contributi sono suddivisi in tre sezioni, che riprendono la scansione enunciata nel titolo del volume. La prima dunque, dedicata ai limiti, indaga quelli "ontologici, bioetici, morali ed estetici che determinano le condizioni di esistenza di un corpo". Vi 
confluiscono nove contributi, a iniziare da quello di Hugo AMARAL, Désirance. The Respiration of the Brossardian corp(s)tex(te) (pp. 19-28), che partendo da una nozione barthesiana enucleata nel Plaisir du texte analizza la scrittura della poetessa Nicole Brossard indirizzata a un corpo utopico, in quanto tale sia limitante che eccessivo e condizione del desiderio. Segue Irene DAL POZ che, in The Practices of Resistence and the Government of Bodies (pp. 29-41), indaga il concetto foucaultiano di resistenza nell'analizzare la depersonalizzazione e la disindividualizzazione del corpo esercitate dal controllo normativo. Nel contributo successivo, Etica della percezione: Bergson e l'immagine-corpo di Andrea NICOLINI (pp. 43-52), è illustrata, in forma speculare, la corporeità da un punto di vista interno, come fonte primaria dell'identità personale $\mathrm{e}$ strumento principale della conoscenza del mondo. Il ricorso a Matière et mémoire di Bergson serve allo studioso per mostrare come lo sforzo del filosofo di unire la tradizione realista e quella idealista lo conduca a una particolare concezione del corpo e alla convinzione che la percezione non possa essere che spuria cioè compromessa con la dimensione emotiva che alberga nella memoria e che viene riattualizzata dal corpo ogni volta che esso percepisce un'immagine. In nome della dimensione multidisciplinare del volume, segue un contributo che analizza questioni etiche legate alla tematica da un punto di vista biomedico, relativamente all'utilizzo di campioni biologici nella ricerca scientifica. Si tratta di Campioni biologici umani e consenso: alcune questioni etiche (pp. 53-66), in cui Chiara MANNELl utilizzando i casi di alcuni "soggetti della ricerca", persone cui sono stati prelevati cellule e tessuti umani, dimostra come lo sviluppo del sapere medico si scontri con i diritti individuali di autodeterminazione e di inviolabilità corporea e dunque come l'innovazione scientifica e tecnologica debbano procedere in osservanza di rigorosi criteri di responsabilità e di interesse pubblico. Manuel Maximilian RIoLo, partendo dal concetto di corpo alterato in accezione digitale, ovvero uscendo dall'universo del reale, applica le Méditations métaphyisiques di Descartes ai videogiochi. Il suo contributo, intitolato Il 'nauta adest navigio' cartesiano e l'avatära' videoludico. Angeli, menti e corpi elettronici nell'epoca dell'incarnazione digitale, illustra come l'unum quid che mantiene unite nel caso dell'essere umano res cogitans e res extensa, mente e corpo, torna a separarsi nel videogioco laddove la mente umana pur calandosi dentro alle spoglie digitali dell'alter ego ne rimane distaccata. Mentre di doppia corporeità del soggetto determinata dalla coesistenza del "corpo vissuto" in quanto nocciolo duro dell'esistenza e del "corpo virtuale" concepito come spazio della soggettivazione si occupa Alessandro FOLADORI in Segno del corpo, corpo del segno. Spinoza e Proust attraverso Deleuze (pp. 79-90). A seguire il contributo di Fabio LIBASCI, Corpo in scena/corpo che inscena. Sadismo e masochismo in Proust (pp. 91-102) che, analizzando alcuni passaggi della Recherche relativi in particolare ai due personaggi di Mlle Vinteuil e Charlus scelti ai due poli estremi dell'opera, riflette sulla natura dell'atto d'amore in essa rappresentato, la cui verità sembra dipendere nel gesto contro il corpo, che sia quello dell'altro o il proprio. E nuovamente su alcune figure di corpi in difetto in particolare di percezione si concentra Alessandra scotTI utilizzando le indagini condotte da Georges Canguilhem sul normale e il patologico di cui propone di cogliere aspetti raramente rilevati ovvero le implicazioni morali ch'esse comportano (Il malato, il mostruoso, l'esausto. A partire dal normale e il patologico in Georges Canguilhem, pp. 103-113). E conclude questa prima parte incentrata sulla nozione di limite - imposto, voluto o oggettivo - il contributo di Lorenzo GRAZIANI, Scrivere con i corpi. Le porno-grafie di Bataille e Gombrowicz tra sovversione del linguaggio e rapporti simulati, pp. 115-125), nel quale viene messa a confronto la funzione assunta dalla metafora pornografica in due testi dei due 
autori letti in parallelo, Histoire de l'œil e Pornografia, alla luce della nozione barthesiana di pornogramma, fusione di discorso e corpo.

4 La seconda sezione passa in rassegna le molteplici costruzioni narrative, filosofiche e letterarie del corpo. Annalisa SACCHI declina il tema all'interno dell'universo teatrale a partire dall'analisi di un passaggio dell'Amleto di Shakespeare, del personaggio beckettiano di Krapp e di una messa in scena del Giulio Cesare da parte della Societas Raffaello Sanzio, allo scopo di indagare il fenomeno di identificazione dello spettatore con l'attore, tramite il richiamo al discorso di Sartre sulla vergogna come forma essenziale del riconoscimento della propria identità attraverso lo sguardo dell'altro (Facci essere umani: sull'empatia, il riconoscimento e la vergogna a teatro, pp. 129-146). Alberto Rossi invece affronta il discorso in ambito romanzesco attraverso l'analisi di tre testi contemporanei ch'egli presenta come autofictions - How Should a Person Be? della canadese Sheila Heti, Troppi paradisi di Walter Siti e Il libro delle parabole dello svedese Per Olov Enquist - caratterizzati dall'assenza del corpo del narratore variamente fagocitato dall'alterità, assenza che l'autore definisce "clamorosa" e che vede strettamente connessa con il genere ibrido praticato nei romanzi in questione (L'assenza del corpo nell'autofiction' contemporanea. Un'indagine comparatistica, pp. 147-157). Francesca DAINESE invece studia corpi non assenti bensì dematerializzati che, inafferrabili, diventano ossessioni: Corpo e identità nell'opera di Patrick Modiano il titolo del contributo (pp. 159-172) in cui è proposta un'interessante veicolazione della sempre evocata quête condotta da Modiano di libro in libro, ricerca di sé e del senso, tramite il tema del corpo fantasmatico, reso tale dalla tragedia dell'Olocausto. Delphine BARRÉ si collega a quest'ultimo tema presentando nel suo contributo alcune opere di testimonianza e alcune produzioni artistiche di donne deportate, mettendo in evidenza come i loro corpi siano al tempo stesso luogo dell'orrore e traccia, memoria dello stesso («They are no longer breasts, they are martyrs!». Women and Their Bodies in the Concentration Camp Experience, pp. 173-186). Segue Francesca FoRCoLIN che studia l'opera di un'autrice contemporanea, Christine Angot, analizzandone la dimensione politica: nel suo studio, Corpo incestuoso, corpo politico nella scrittura di Christine Angot (pp. 187-197) è illustrata una strategia narrativa che tramite l'invasione della pagina da parte del corpo, della sua figura violata, vuole significare la valenza simbolica dell'atto incestuoso il quale, fisicamente perpetrato ma insieme connesso testualmente con figure mitiche che lo evocano, instaura un contratto sociale basato sul sopruso, sopruso contro il quale Angot scaglia la forza del suo linguaggio. Giulia ARAGONESE a sua volta indaga dimensione sociale e habitus del corpo in un contributo che riprende il testo di una conferenza del 1934 di Marcel Mauss a lungo trascurata nella quale lo studioso analizzava le differenti tecniche del corpo che gli individui mettono a punto in base alla tradizione di riferimento per operare nel loro specifico contesto culturale e geografico (Marcel Mauss $e$ "Les techinques du corps". Sollecitazioni sul corpo tra tecnica, aneddotica e cinema, pp. 199-214). Mentre Lorenzo VITALE, con Il corpo: il mondo. Meditazioni sull'inconscio leibniziano (pp. 215-228), mette in evidenza come l'esistenza possa riassumersi in una "totalità interconnessa" tra corpo, inconscio e mondo. E Giulia BIASCI infine studia la sintomatologia di malattie che affliggono la sfera morale e fisica insieme, sintomatologia considerata come linguaggio del corpo e centrale in numerose opere di Diderot, interpretata come conseguenza di un sostanziale dissidio tra il corpo e la mente, la ragione e la passione, le norme imposte dall'ordine sociale e il desiderio 
pulsionale di trasgredire (Rappresentare il corpo e le sue patologie nel Settecento francese: la sfida dello scrittore e del medico, pp. 229-241).

5 La terza e ultima sezione è dedicata poi alle intensità del corpo, "sublimate nella loro traslitterazione teatrale, enigmistica, cinematografica e fisiologica" come spiegano $\mathrm{i}$ curatori. Il taglio in questo caso è più ordinatamente diacronico, il primo contributo di Rosanna ROTA offre infatti un interessante studio sulle cicatrici come esempio di scrittura sul corpo nel mondo latino, a partire dallo Pseudolus di Plauto, opera in cui il segno sulla pelle è marchio d'infamia per lo schiavo ma al tempo stesso medaglia al valore per il cittadino-soldato che, esibendo le sue cicatrici sul petto, dimostra di non essere fuggito di fronte al nemico ('Tergum cicatricosum'. Un esempio di costruzione culturale del corpo nel mondo plautino, pp. 245-256). Segue Giulia FARINA che, in Procedimenti di anatomia enigmistica: il corpo e le sue parti nell'indovinello antico e medievale (pp. 257-270), studia la presenza del corpo nel linguaggio umano tramite l'analisi di indovinelli costruiti sfruttando la porosità del lessico relativo al corpo. Flavia PALMA poi analizza il meccanismo speculare di beffa e contro-beffa in una novella del Bandello mettendo in luce come il corpo sia allo stesso tempo terreno di lotta e strumento di potere nel rapporto tra due amanti (Il corpo tra voyeurismo e sadica vendetta in Matteo Bandello. Considerazioni sulla novella I, 3, pp. 271-281). Elisa DESTRO analizza le fantasie oniriche di Franz Kafka tratte dalle sue annotazioni di sogni, mostrando come in questi ultimi i corpi torturati e deformi siano metafora di una parola dannata (Il piacere della tortura. Fantasie masochistiche, corpi sofferenti e metamorfosi nei sogni di Franz Kafka, pp. 283-295). I due contributi che seguono, quello di Lisann ANDERS (Trapped in the Body: the Fragmentation of Body and Mind in Chuck Palahniuk's "Fight Club", pp. 297-309) e quello di Francesco DALL'OLIO ("Don't dream it, be it»: esaltazione e distruzione del corpo fra "Baccanti” $e$ "The Rocky Horror Picture Show", pp. 311-322), analizzano il rapporto tra corpo e identità la prima rileggendo un romanzo di Chuck Palahniuk in relazione alla sua trasposizione cinematografica, nella prospettiva dell'annientamento del corpo necessario come segno, ma anche come sintomo della crisi del soggetto moderno; il secondo interrogandosi sul concetto di "dionisiaco" riferito a opere considerate liberatorie rispetto agli impulsi repressi della società, tramite un parallelo tra le Baccanti di Euripide e il film di Jim Sharman, versione cinematografica del celebre musical di Richard O'Brien. A chiusura della sezione e del volume, la componente più fisiologica del corpo. Traianos MANOs, in 'Ars scatologica'. Appunti sulla letteratura scatologica ovvero Verso una filologia degli escrementi (pp. 323-337) offre una panoramica della letteratura scatologica da Aristofane a Ceronetti, passando per Giovenale, la polemica anticattolica in ambito luterano, Joyce, Pasolini e Zanzotto, non dimenticando Pietro Manzoni e il saggio di Bakhtin su Rabelais e proponendo interessanti possibili sviluppi delle sue riflessioni. Mentre Lorena CARRARA, in Bocca, buchi, confini e alterità (pp. 339-353), utilizzando testi di natura diversa, investiga il tema della bocca intesa come limen. La studiosa sottolinea come tutti i buchi del corpo possano essere letti come soglie tra il mondo interno e l'esterno, ma come la bocca in particolare sia il locus di una duplice oralità, quella del cibo e della parola, entrambi strumenti di incorporazione dell'altro.

Il corpo descritto dagli autori del volume è insomma un insieme "di caratteristiche, accidenti e simbolizzazioni che si incontrano al crocevia dell'individuale e del collettivo", insieme irriducibile e perennemente inconoscibile nella sua complessità e proprio per questo tanto più adeguato a indagini interdisciplinari come quelle proposte. 